\title{
Dynamics of a Guanaco-sheep Competitive System with Unilateral and Bilateral Control
}

Jing Xu

Xinyang Normal University

Mingzhan Huang

Xinyang Normal University

Xinyu Song ( $\square$ xysong88@163.com )

Xinyang Normal University

\section{Research Article}

Keywords: Guanaco-sheep competitive model, Successor function, Periodic solution, Existence and stability

Posted Date: July 19th, 2021

DOI: https://doi.org/10.21203/rs.3.rs-691485/v1

License: (c) (i) This work is licensed under a Creative Commons Attribution 4.0 International License. Read Full License

Version of Record: A version of this preprint was published at Nonlinear Dynamics on January 24th, 2022. See the published version at https://doi.org/10.1007/s11071-021-07128-1. 


\title{
Dynamics of a guanaco-sheep competitive system with unilateral and bilateral control
}

\author{
Jing Xu • Mingzhan Huang • Xinyu Song
}

Received: date / Accepted: date

\begin{abstract}
In this paper, based on a guanaco-sheep competitive system, we develop and analyze mathematical models with unilateral and bilateral control for the management of overgrazing. We first analyze the dynamics of the uncontrolled system. Then, we investigate the system with impulsive control by differential equation geometry theory. And we mainly prove the existence and stability of order-1 periodic solution for unilateral control system and order-2 periodic solution for bilateral control system. Comparing the unilateral and bilateral control strategy, we encourage bilateral control rather than unilateral control for the management of sheep species.
\end{abstract}

Keywords Guanaco-sheep competitive model; Successor function; Periodic solution; Existence and stability

\section{Introduction}

The guanaco which distributed in the arid and semiarid areas 1], is a native and wild camelid species in South America [2], and the population size of this species has suffered a sustained decline. Authors of 3 showed that the guanacos occupy

Jing $\mathrm{Xu}$

School of Mathematics and Statistics, Xinyang Normal University, Xinyang 464000, China

Mingzhan Huang

School of Mathematics and Statistics, Xinyang Normal University, Xinyang 464000, China

Xinyu Song (Corresponding author)

School of Mathematics and Statistics, Xinyang Normal University, Xinyang 464000, China

E-mail: xysong88@163.com 
only $26 \%$ of its original scope. And the results of 4 suggested that the range distribution of the guanacos has been reduced by $60 \%$ in Argentina, $75 \%$ in Chile. This phenomenon is mainly attributed to habitat change and loss, competition with livestock (sheep, cattle and horses) for pasture, and the lack of a proper management for livestock [5].

Literature study shows that there is a competitive relationship between the guanaco and sheep species. Puig et al. 6] indicated that competition between guanaco and domestic animals is due to the fact that their diets overlap up to $83 \%$. Baldi et al. [7] and Gordon [8] suggested that guanaco and sheep compete for food and water resources. Ranchers believe that guanaco depletes food supplies, hence reducing productivity of sheep species 9. So it is very meaningful to investigate the interaction of these two species. In this regard, Laguna 10] developed a three-dimensional mathematical model with two competing herbivores (sheep and guanaco) and one predator, and analyzed the steady state of the occupation of patches for the three species. Daza et al. 11] proposed a delayed differential model capturing the interaction between sheep and guanaco, and investigated coexistence and extinction of these two species.

However, overgrazing or domestic animal overstock has caused vegetation degradation, and the reduction of wildlife is the most serious consequences of vegetation degradation. Pedrana et al. 1] indicated that sheep species seems to have a higher impact on vegetation. Marino et al. 12] showed that grassland degradation and the decline of guanaco species will continue due to overgrazing. Bharucha et al. 13 . pointed out that overgrazing modified the plant cover and impaired its productive capacity. So what management programs should be developed by human beings to change this situation and protect guanaco species and grassland? With human intervention, the population size of sheep and guanaco species will change in a short time, and this phenomenon could be well described by impulsive differential equation [14] which has been extensively applied in applications 15 27]. For example, integrated pest management models were studied in $15-17$ where the existence of order-1 periodic solution is proved, and the authors of [18 and [19] also considered the optimal control problem. For resource management, Guo et al. 22 analyzed a algae-fish with unilateral control. And bilateral control strategy is applied in $23,25,27$, where the existence and stability of order-2 periodic solution are mainly proved. And Huang et al. 27. also analyzed the attraction region of the system, and performed bifurcation diagrams of periodic solution. In addition, bilateral control strategy has also been applied to biodiversity conservation and commodity price adjustment $[24,26]$. 
Motivated by the above research, in this paper, by constructing semi-continuous dynamical systems, we develop mathematical models for the management of sheep and guanaco species. On the one hand, the number of sheep species will be limited to avoid overgrazing; on the other hand, the economic interests of farmers should also be protected while keeping sheep from overgrazing. Based on these two aspect$\mathrm{s}$, we consider unilateral and bilateral control strategies to keep the sheep species in a suitable range.

The rest of the paper is organised as follows. In section 2, we develop guanacosheep competitive systems with unilateral and bilateral control strategy. In section 3 the existence of the order-1 and order-2 periodic orbit is proved by successor function. And stability of the order-1 and order- 2 periodic orbit is verified by analogue of the Poincaré criterion. In section 4 the numerical simulations are carried out with a special case to validate the theoretical results. Finally, a brief conclusion is presented in section 5 .

\section{Model formulation}

For the guanaco and sheep species, their competitive ability is related to their body size. Tilman et al. 28] considered that the competitiveness of these two competing herbivores is not in the same hierarchi. And Daza et al. 11] pointed out that there exists hierarchical competition between guanaco and sheep, the guanaco is assumed to be the superior species and sheep is the inferior one. So we assume that the effect of inferior species on superior species satisfies Holling II functional response, and the influence of higher competitors on lower competitors satisfies a linear function. Based on the classical two-species competitive system [29], we get a modified competitive system as follows:

$$
\left\{\begin{array}{l}
\frac{\mathrm{d} x}{\mathrm{~d} t}=x\left(a-b x-\frac{c y}{1+y}\right) \\
\frac{\mathrm{d} y}{\mathrm{~d} t}=y(d-e x-f y)
\end{array}\right.
$$

where $x(t)$ and $y(t)$ stand for the density of guanaco and sheep species, respectively. The parameters $a, b, c, d, e$ and $f$ have the same biological meaning as in 29].

As the application of semi-continuous dynamic system in overgrazing management, we mainly investigate guanaco-sheep competitive systems with unilateral and bilateral control strategies. On the one hand, desertification caused by overgrazing should be gradually mitigated; on the other hand, the interests of farmers should also be protected. So we are committed to keeping the number of sheep 
within a reasonable range $\left[h_{1}, h_{2}\right]$. To this end, we extend system (1) by incorporating two control thresholds $y=h_{1}$ and $y=h_{2}$. When the number of sheep species decreases to $H_{1}$, herdsman can increase the number of sheep by a certain proportion, while reducing the number of original alpacas by a certain amount. When the quantity of sheep species reaches to $h_{2}$, people can release guanaco and harvest sheep suitably. Thus, two guanaco-sheep competitive systems with unilateral control are established as follows:

$$
\left\{\begin{aligned}
\frac{\mathrm{d} x}{\mathrm{~d} t} & =x\left(a-b x-\frac{c y}{1+y}\right) \\
\frac{\mathrm{d} y}{\mathrm{~d} t} & =y(d-e x-f y) \\
\Delta x & =-\tau_{1}, \Delta y=\alpha_{1} y
\end{aligned}\right\} \quad y=h_{1},
$$

and

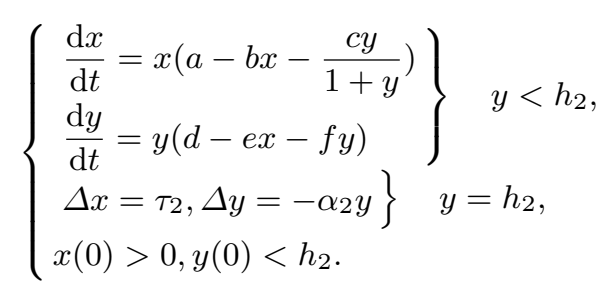

When two kinds of control are considered simultaneously, we have the following competitive system with bilateral control:

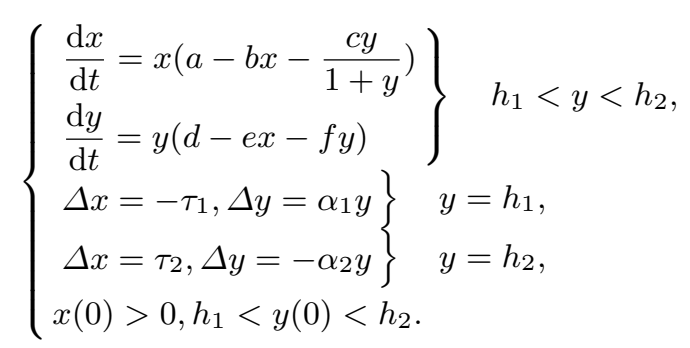

All the parameters in systems (2), (3) and (4) are positive, and $0<\alpha_{1}, \alpha_{2}<1$.

\section{Main results}

In this section, we mainly discuss the dynamical properties of system (1) and the existence and stability of periodic solution of systems (2), (3) and (4). 
3.1 Dynamics of free system (1)

For system (1), it is easy to determine that trivial equilibrium $O(0,0)$ is always unstable. And the equilibrium $E_{1}\left(\frac{a}{b}, 0\right)$ is locally stable if $a e>b d$, otherwise it is unstable. The equilibrium $E_{2}\left(0, \frac{d}{f}\right)$ is locally stable if $a(f+d)>c d$, otherwise it is unstable. Positive equilibrium $\left(x^{*}, y^{*}\right)$ satisfies the following equation:

$$
\left\{\begin{array}{l}
a-b x-\frac{c y}{1+y}:=f(x, y) \\
d-e x-f y:=g(x, y)
\end{array}\right.
$$

After simple calculation, we have $x^{*}=\frac{d-f y^{*}}{e}$, and $y^{*}$ satisfies the following second-order algebraic equation:

$$
a_{1} y^{2}+b_{1} y+c_{1}=0
$$

where $a_{1}=b f, b_{1}=b(f-d)+e(a-c), c_{1}=a e-b d$. Define $\Delta=b_{1}^{2}-4 a_{1} c_{1}$.

Obviously, the curve $l_{2}:=(x, y) \mid f(x, y)=0$ has a horizontal asymptote $y=$ -1 and a vertical asymptote $y=\frac{a-c}{b}$, respectively. Combining with differential equation geometry theory, we analyze the existence and stability of the positive equilibrium of system (1) from the following two cases.

Case 1. If $a>c$, then vertical asymptote $y=\frac{a-c}{b}$ lies on the right of $y$-axis. The location of the two isoclines $l_{1}$ and $l_{2}$ is shown in Figure 1 .

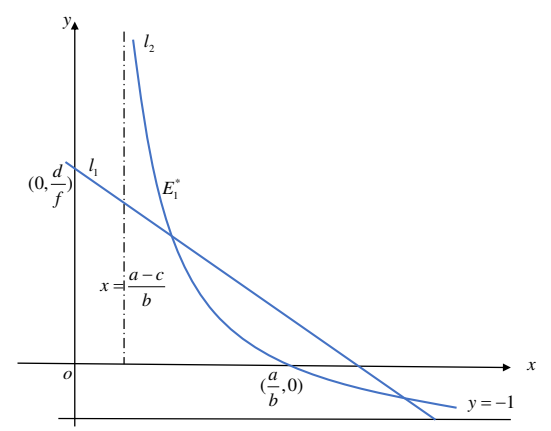

(a)

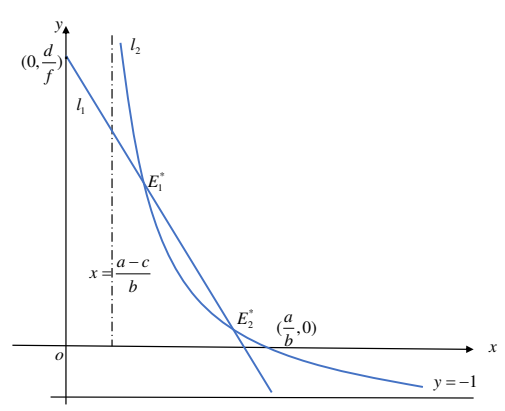

(b)

Fig. 1 The location of the two isoclines $l_{1}$ and $l_{2} a>c$.

1. If $\Delta>0, a>c, a e<b d, b(f-d)+e(a-c)<0, a(d+f)>c d$, system (1) exists unique positive equilibrium $E_{1}^{*}\left(x_{1}^{*}, y_{1}^{*}\right)$ (see Figure $1(\mathrm{a})$. The Jacobian 
matrix of system (1) evaluated at $E_{1}^{*}$ is

$$
J_{E_{1}^{*}}=\left(\begin{array}{cc}
-b x_{1}^{*} & -\frac{c x_{1}^{*}}{\left(1+y_{1}^{*}\right)^{2}} \\
-e y_{1}^{*} & -f y_{1}^{*}
\end{array}\right)
$$

Since $-\frac{f_{x}\left(x_{1}^{*}, y_{1}^{*}\right)}{f_{y}\left(x_{1}^{*}, y_{1}^{*}\right)}<-\frac{g_{x}\left(x_{1}^{*}, y_{1}^{*}\right)}{g_{y}\left(x_{1}^{*}, y_{1}^{*}\right)}$, i.e., $b f>\frac{c e}{\left(1+y_{1}^{*}\right)^{2}}$, then we have

$$
\begin{aligned}
\operatorname{Tr}\left(J_{E_{1}^{*}}\right) & =-\left(b x_{1}^{*}+f y_{1}^{*}\right)<0 ; \\
\operatorname{det}\left(J_{E_{1}^{*}}\right) & =x_{1}^{*} y_{1}^{*}\left(b f-\frac{c e}{\left(1+y_{1}^{*}\right)^{2}}\right)>0 .
\end{aligned}
$$

So $E_{1}^{*}$ is locally stable. By constructing Dulac function $D(x, y)=\frac{1}{x y}$, we find that system (1) has no limit cycle. So we conclude that $E_{1}^{*}$ is globally stable.

2. If $\Delta>0, a>c, a e>b d, b(f-d)+e(a-c)<0, a(d+f)>c d$, system (1) has two positive equilibria $E_{1}^{*}\left(x_{1}^{*}, y_{1}^{*}\right)$ and $E_{2}^{*}\left(x_{2}^{*}, y_{2}^{*}\right)$ (see Figure 1(b). Similarly, we can conclude that $E_{1}^{*}\left(x_{1}^{*}, y_{1}^{*}\right)$ is locally stable, and $E_{2}^{*}\left(x_{2}^{*}, y_{2}^{*}\right)$ is a saddle point.

Case 2. If $a<c$, then vertical asymptote $y=\frac{a-c}{b}$ lies on the left of $y$-axis. The location of the two isoclines $l_{1}$ and $l_{2}$ is shown in Figure 2 .

1. If $\Delta>0, a<c, b(f-d)+e(a-c)<0, a(d+f)<c d$, ae $>b d$, system (1) exists unique positive equilibrium $E_{1}^{*}\left(x_{1}^{*}, y_{1}^{*}\right)$ (see Figure 2(a) . It is easy to determine that $E_{1}^{*}\left(x_{1}^{*}, y_{1}^{*}\right)$ is unstable.

2. If $\Delta>0, a<c, b(f-d)+e(a-c)>0, a(d+f)>c d, a e<b d$, system (1) exists unique positive equilibrium $E_{1}^{*}\left(x_{1}^{*}, y_{1}^{*}\right)$ (see Figure 2(b)]. It is easy to determine that $E_{1}^{*}\left(x_{1}^{*}, y_{1}^{*}\right)$ is globally stable.

3. If $\Delta>0, a<c, b(f-d)+e(a-c)<0, a e>b d, a(d+f)>c d$, system (1) has two positive equilibria $E_{1}^{*}\left(x_{1}^{*}, y_{1}^{*}\right)$ and $E_{2}^{*}\left(x_{2}^{*}, y_{2}^{*}\right)$ (see Figure 2(c)]. We can conclude that $E_{1}^{*}\left(x_{1}^{*}, y_{1}^{*}\right)$ is locally stable, and $E_{2}^{*}\left(x_{2}^{*}, y_{2}^{*}\right)$ is a saddle point.

3.2 Existence and stability of the order-1 periodic solution

In this work, we mainly prove the existence and stability of periodic solution when the unique positive equilibrium $E_{1}^{*}\left(x_{1}^{*}, y_{1}^{*}\right)$ is a saddle point. It then follows that the proof the existence of order-1 periodic solution of system (2).

\subsubsection{Existence of the order-1 periodic solution of system [2]}

As is shown in Figure 3 , the dashed lines indicate the separatrix of saddle point which divide the first quadrant into four regions. Line $l_{3}$ intersects with the phase 


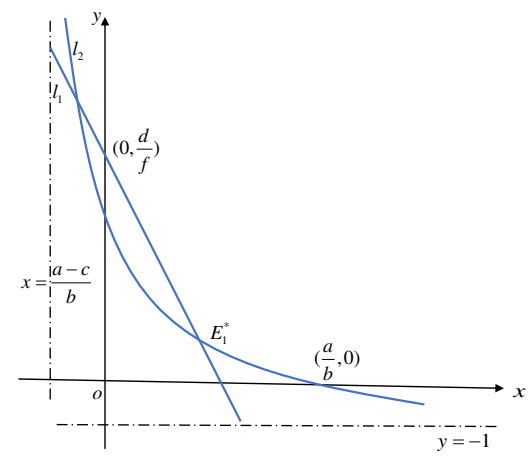

(a)

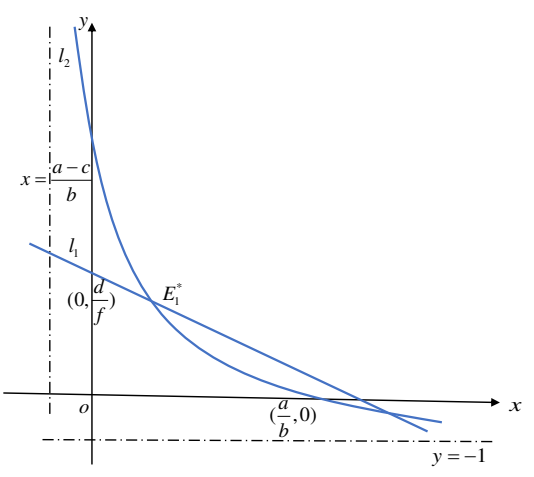

(b)

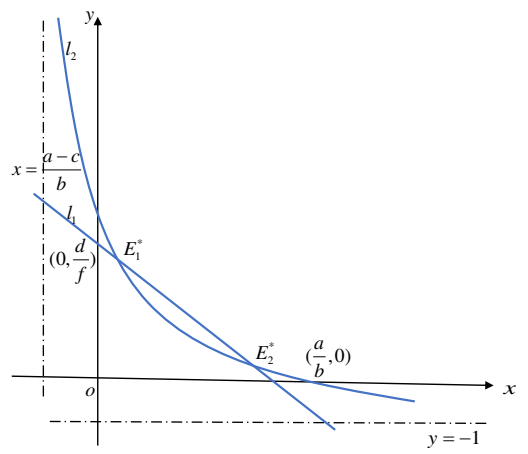

(c)

Fig. 2 The location of the two isoclines $l_{1}$ and $l_{2}$ for $a<c$.

set $N_{1}=\left\{(x, y) \in R_{+}^{2} \mid y=\left(1+\alpha_{1}\right) h_{1}\right\}$ and impulsive set $M_{1}=\left\{(x, y) \in R_{+}^{2} \mid y=\right.$ $\left.h_{1}\right\}$ at point $A_{2}$ and $B_{2}$, respectively. We denote the coordinates of points $A_{2}$ and $B_{2}$ as $\left(x_{A_{2}},\left(1+\alpha_{1}\right) h_{1}\right)$ and $\left(x_{B_{2}}, h_{1}\right)$. We now give the existence of the order-1 periodic solution of system 2 .

Theorem 1 If $x_{A_{2}}<x_{B_{2}}-\tau_{1}$, then system (2) exists an order-1 periodic solution.

Proof Denote the intersection point of the curve $l_{2}$ and the line $M_{1}$ by $B_{1}$. According to the phase diagram of system (2), there must exist a point $A_{1}\left(x_{A_{1}}, h_{1}\right) \in N_{1}$ such that the trajectory from $A_{1}$ intersects with $M_{1}$ at point $B_{1}$, then jumps to $A_{1}^{+}$due to the impulsive effect. Obviously, $x_{A_{1}^{+}}<x_{B_{1}}<x_{A_{1}}$, so we have that the successor function of point $A_{1}$ satisfies that $G\left(A_{1}\right)=x_{A_{1}^{+}}-x_{A_{1}}<0$. Thus, we only need to find another point $A_{2} \in N_{1}$ such that $G\left(A_{2}\right)>0$. Consider the intersection point $A_{2}$ of the dashed line $l_{3}$ and $N_{1}$, the trajectory from $A_{2}$ intersects with $N_{1}$ at point $B_{2}$, and then jumps to $A_{2}^{+}$due to the impulsive effect. Based 
on the condition $x_{A_{2}}<x_{B_{2}}-\tau_{1}$, we have that $G\left(A_{2}\right)=x_{B_{2}}-\tau_{1}-x_{A_{2}}>0$, as is shown in Figure 4. According to the continuity of successor functions, there must exist a point $A \in N_{1}$ between $A_{1}$ and $A_{2}$ satisfying that $G(A)=0$, i.e., the trajectory $\widehat{A B} \cup \overline{B A}$ forms an order-1 periodic solution of system (2).

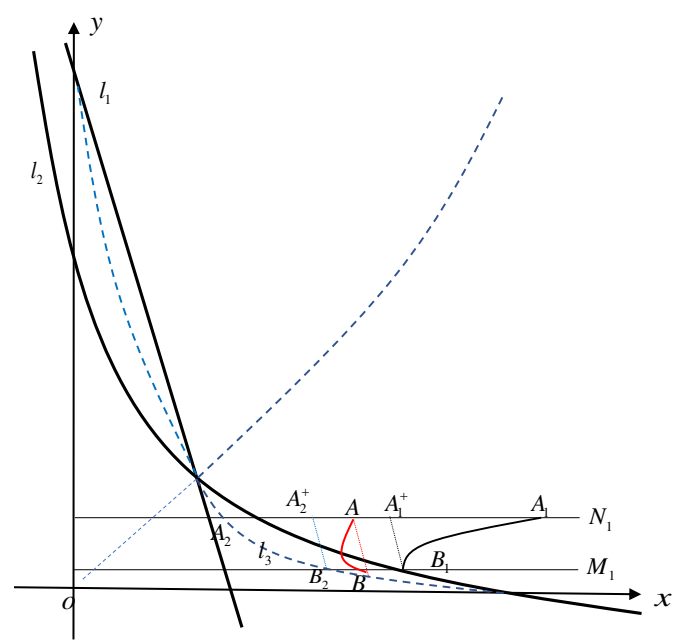

Fig. 3 The existence of order-1 periodic solution of system 2 .

It then follows the proof of the stability of order- 1 periodic solution of system (2).

3.2.2 Stability of order-1 periodic solution of system (2)

Assume that $T_{1}(t)=\left(\xi_{1}(t), \eta_{1}(t)\right)$ is the order-1 periodic solution of system (2) with period $T_{1}$. Define $\xi_{1}(0)=x_{A}, \eta_{1}(0)=y_{A}, \xi_{1}\left(T_{1}\right)=x_{B}, \eta_{1}\left(T_{1}\right)=y_{B}=h_{1}$, $\xi_{1}\left(T_{1}+0\right)=x_{B}-\tau_{1}=x_{A}, \eta_{1}\left(T_{1}+0\right)=\left(1+\alpha_{1}\right) h_{1}=y_{A}$.

Theorem 2 The order-1 periodic solution of system (2) is orbitally asymptotically stable if $\left|\Theta_{1}\right|<1$ holds, where

$$
\Theta_{1}=\frac{x_{B}\left(d-e\left(x_{B}-\tau_{1}\right)-f h_{1}\left(1+\alpha_{1}\right)\right)}{\left(d-e x_{B}-f h_{1}\right)\left(x_{B}-\tau_{1}\right)} .
$$

Proof

$$
\begin{aligned}
& P(x, y)=x\left(a-b x-\frac{c y}{1+y}\right), Q(x, y)=y(d-e x-f y), \frac{\partial P}{\partial x}=a-2 b x-\frac{c y}{1+y}, \\
& \frac{\partial Q}{\partial y}=d-e x-2 f y, E(x, y)=-\tau_{1}, F(x, y)=\alpha_{1} y, \varphi(x, y)=y-h_{1}, \\
& \frac{\partial E}{\partial x}=0, \frac{\partial E}{\partial y}=0, \frac{\partial F}{\partial x}=0, \frac{\partial F}{\partial y}=\alpha_{1}, \frac{\partial \varphi}{\partial x}=0, \frac{\partial \varphi}{\partial y}=1 .
\end{aligned}
$$


By simple calculation, we have

$$
\begin{aligned}
\Delta_{1} & =\frac{P_{+}\left(\frac{\partial F}{\partial y} \frac{\partial \varphi_{1}}{\partial x}-\frac{\partial F}{\partial x} \frac{\partial \varphi}{\partial y}+\frac{\partial \varphi}{\partial x}\right)+Q_{+}\left(\frac{\partial E}{\partial x} \frac{\partial \varphi_{1}}{\partial y}-\frac{\partial E}{\partial y} \frac{\partial \varphi}{\partial x}+\frac{\partial \varphi}{\partial y}\right)}{P\left(\frac{\partial \varphi}{\partial x}\right)+Q\left(\frac{\partial \varphi_{1}}{\partial y}\right)} \\
& =\frac{Q_{+}\left(\xi_{1}(T+0), \eta_{1}(T+0)\right)}{Q\left(\xi_{1}(T+0), \eta_{1}(T+0)\right)}=\frac{\left(1+\alpha_{1}\right)\left(d-e\left(x_{B}-\tau_{1}\right)-f h_{1}\left(1+\alpha_{1}\right)\right)}{d-e x_{B}-f h_{1}},
\end{aligned}
$$

and

$$
\begin{aligned}
\int_{0^{+}}^{T_{1}}\left(\frac{\partial P}{\partial x}+\frac{\partial Q}{\partial y}\right) d t & =\int_{0^{+}}^{T_{1}} a-2 b x-\frac{c y}{1+y}+d-e x-2 f y d t \\
& =\int_{x_{A}}^{x_{B}} \frac{1}{x} d t+\int_{y_{A}}^{h 1} \frac{1}{y} d t-\int_{0^{+}}^{T_{1}} b x+f y d t \\
& =\ln \frac{x_{B}}{x_{A}}+\ln \frac{h_{1}}{y_{A}}-\int_{0^{+}}^{T_{1}} b x+f y d t
\end{aligned}
$$

Then we have

$$
\begin{aligned}
\mu_{2} & =\Delta_{1} \exp \left\{\int_{0^{+}}^{T_{1}}\left(\frac{\partial P}{\partial x}+\frac{\partial Q}{\partial y}\right) d t\right\} \\
& =\frac{\left(1+\alpha_{1}\right)\left(d-e\left(x_{B}-\tau_{1}\right)-f h_{1}\left(1+\alpha_{1}\right)\right)}{d-e x_{B}-f h_{1}} \frac{x_{B}}{x_{A}} \frac{h_{1}}{y_{A}} \exp \left\{-\int_{0^{+}}^{T_{1}} b x+f y d t\right\} \\
& =\frac{\left(d-e\left(x_{B}-\tau_{1}\right)-f h_{1}\left(1+\alpha_{1}\right)\right)}{d-e x_{B}-f h_{1}} \frac{x_{B}}{x_{B}-\tau_{1}} \exp \left\{-\int_{0^{+}}^{T_{1}} b x+f y d t\right\} .
\end{aligned}
$$

Obviously, $0<\exp \left\{-\int_{0^{+}}^{T_{1}}(b x+f y) d t\right\}<1$, if $\Theta_{1}=\left|\frac{x_{B}\left(d-e\left(x_{B}-\tau_{1}\right)-f h_{1}\left(1+\alpha_{1}\right)\right)}{\left(d-e x_{B}-f h_{1}\right)\left(x_{B}-\tau_{1}\right)}\right|<$ 1 , then the order-1 periodic solution is orbitally asymptotically stable.

\subsubsection{Existence and stability of the order-1 periodic solution of system (3)}

Next, we mainly investigate the existence of the order-1 periodic solution of system (3).

As is shown in Figure 4, line $l_{4}$ intersects with the phase set $N_{2}=\{(x, y) \in$ $\left.R_{+}^{2} \mid y=\left(1-\alpha_{2}\right) h_{2}\right\}$ and impulsive set $M_{2}=\left\{(x, y) \in R_{+}^{2} \mid y=h_{2}\right\}$ at points $A_{4}$ and $B_{4}$, respectively. We denote the coordinates of points $A_{4}$ and $B_{4}$ as $\left(x_{A_{4}},(1-\right.$ $\left.\left.\alpha_{2}\right) h_{2}\right)$ and $\left(x_{B_{4}}, h_{2}\right)$. In the following, we prove the existence of order-1 periodic solution of system (3).

Theorem 3 If $x_{B_{4}}+\tau_{2}<x_{A_{4}}$, then system (3) exists an order-1 periodic solution.

Proof Select a point $A_{3}\left(x_{A_{3}},\left(1+\alpha_{2}\right) h_{2}\right)$ in phase set $N_{2}$ where $x_{A_{3}}$ is sufficiently small, the trajectory from $A_{3}$ intersects with $M_{2}$ at point $B_{3}$, and then jumps to $A_{3}^{+}$due to the impulsive effect. Since $x_{A_{3}}$ is sufficiently small, then the the successor function of point $A_{3}$ satisfies that $G\left(A_{1}\right)=x_{A_{3}^{+}}-x_{A_{3}}>0$. Next, 
we only need to find another point $A_{4} \in N_{2}$ such that $G\left(A_{4}\right)<0$. Consider the intersection point $A_{4}$ of the dashed line $l_{4}$ and $N_{2}$, the trajectory from $A_{4}$ intersects with $M_{2}$ at point $B_{4}$, and then jumps to $A_{4}^{+}$due to the impulsive effect. Since $x_{B_{4}}+\tau_{2}<x_{A_{4}}$, we get $G\left(A_{4}\right)=x_{B_{4}}+\tau_{2}-x_{A_{4}}<0$, as is shown in Figure 4. By the continuity of successor function, there must exist a point $A$ between $A_{3}$ and $A_{4}$ satisfying that $G(A)=0$, i.e., there exist an order-1 periodic solution of system (3).

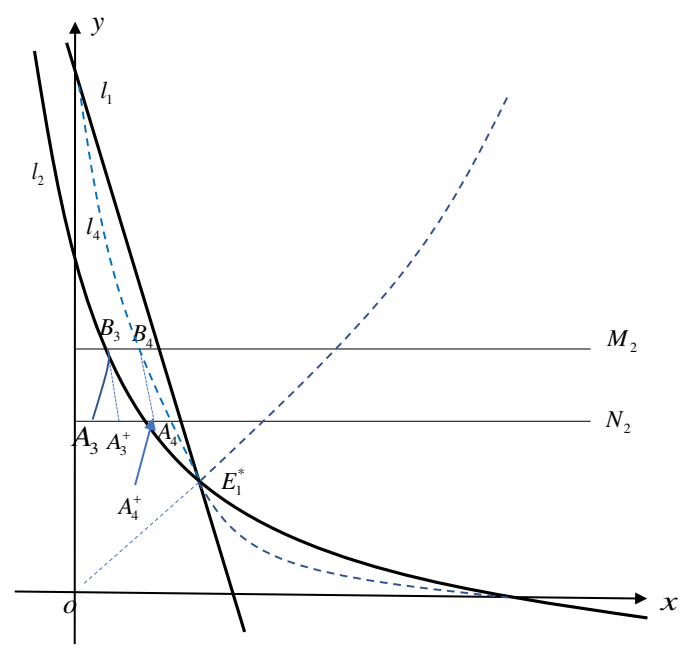

Fig. 4 The existence of order-1 periodic solution of system (3).

Remark 1 The proof of the stability of order-1 periodic solution of system (3) is similar to the proof of theorem 2 , so we omit it here.

3.3 Existence and stability of the order-2 periodic solution

In this subsection, we mainly prove the existence and stability of order-2 periodic solution of system (4).

3.3.1 Existence of the order-2 periodic solution of system (4)

Next, we will prove the existence of order-2 periodic solution of system (4). For convenience, we suppose that the isocline $l_{1}$ intersects with impulsive set $M_{2}$ and phase set $N_{1}$ at points $G_{1}\left(x_{G_{1}}, h_{2}\right)$ and $G_{2}\left(x_{G_{2}},\left(1+\alpha_{1}\right) h_{1}\right)$, respectively. Isocline $l_{2}$ and the line $l_{3}$ intersect with impulsive set $M_{1}$ at point $D_{3}\left(x_{D_{3}}, h_{1}\right)$ 
and point $H\left(x_{H}, h_{1}\right)$, respectively. $l_{5}$ and $l_{6}$ intersect with $N_{1}$ and $N_{2}$ at point $F_{1}\left(x_{F_{1}},\left(1-\alpha_{2}\right) h_{2}\right), F_{2}\left(x_{F_{2}},\left(1+\alpha_{1}\right) h_{1}\right)$, respectively. Besides, there must exist a point $S\left(x_{S},\left(1-\alpha_{2}\right) h_{2}\right) \in N_{2}$, such that the trajectory from point $S$ intersects with impulsive set $M_{1}$ at point $D_{3}$.

Theorem 4 If $x_{D_{3}}-x_{F_{2}}<\tau_{1}<x_{H}, x_{F_{1}}<\tau_{2}<x_{S}-x_{G_{1}}$, then system (4) has an order-2 periodic solution.

Proof Choose a point $A_{1} \in N_{1}$, where $A_{1}$ is very closed to $y$-axis, i.e., $x_{A_{1}}$ is positive and small enough. The trajectory starting from point $A_{1}$ will intersect with the impulsive set $M_{2}$ at point $B_{1}\left(x_{B_{1}}, h_{2}\right)$. Based on the second impulsive function, point $B_{1}$ is mapped to point $C_{1}\left(x_{C_{1}},\left(1-\alpha_{a}\right) h_{2}\right) \in N_{2}$, where $x_{C_{1}}=x_{B_{1}}+\tau_{2}$. Due to that $x_{F_{1}}<\tau_{2}<x_{S}-x_{G_{1}}$, so $x_{F_{1}}<x_{C_{1}}<x_{S}$. The trajectory starting from point $C_{1}$ will intersect with the impulsive set $M_{1}$ at point $D_{1}\left(x_{D_{1}}, h_{1}\right)$, then $D_{1}$ is on the right side of the line $l_{3}$. Based on the first impulsive function, point $D_{1}$ is mapped to point $A_{1}^{+} \in N_{1}$, where point $A_{1}^{+}$is the order-2 successor point of $A_{1}$, and $x_{A_{1}^{+}}=x_{D_{1}}-\tau_{1}$. Since $x_{D_{3}}-x_{F_{2}}<\tau_{1}<x_{H}<x_{D_{1}}$, we have $0<x_{A_{1}^{+}}<x_{D_{1}}-\left(x_{D_{3}}-x_{F_{2}}\right)<x_{F_{2}}$. In addition, $x_{A_{1}}$ is positive and small enough, so we have $G\left(A_{1}\right)=x_{A_{1}^{+}}-x_{A_{1}}>0$ (see Figure $5(\mathrm{a})$.

Choose another point $A_{2} \in N_{1}$, and $A_{2}$ is sufficiently closed to point $F_{2}$ and it is on the left side of point $F_{2}$. Similar to the discussion above, the trajectory starting from point $A_{2}$ intersects with $M_{2}$ at point $B_{2}\left(x_{B_{2}}, h_{1}\right)$, where $x_{B_{2}}>$ $x_{B_{1}}$. Then point $B_{2}$ is mapped to point $C_{2}\left(x_{C_{2}},\left(1-\alpha_{2}\right) h_{2}\right) \in N_{2}$, and we have $x_{F_{1}}<x_{C_{1}}<x_{C_{2}}<x_{S}$. The trajectory starting from $C_{2}$ intersects with impulsive set $M_{1}$ at point $D_{2}\left(x_{D_{2}}, h_{1}\right)$ satisfying that $x_{H}<x_{D_{1}}<x_{D_{2}}<x_{D_{3}}$. Then point $D_{2}$ is mapped to $A_{2}^{+} \in N_{1}$, where $x_{A_{2}^{+}}=x_{D_{2}}-\tau_{2}$, thus $0<x_{A_{2}^{+}}<$ $x_{D_{2}}-\left(x_{D_{3}}-x_{F_{2}}\right)<x_{F_{2}}$. Since $A_{2}$ is sufficiently closed to $F_{2}$, we have $x_{A_{2}^{+}}<x_{A_{2}}$, i.e. $G\left(A_{2}\right)=x_{A_{2}^{+}}-x_{A_{2}}<0$. By the continuity of successor function, there is a point $A$ between $A_{1}$ and $A_{2}$ satisfying $G(A)=0$, then $\widehat{A B} \cup \overline{B C} \cup \widehat{C D} \cup \overline{D A}$ forms an order-2 periodic solution, as is shown in Figure $5(\mathrm{~b})$.

It then follows that the stability of order-2 periodic solution of system (4).

\subsubsection{Stability of the order-2 periodic solution}

Let $T(t)=(\xi(t), \eta(t))$ be the order-2 periodic solution of system (4). Define $\xi(0)=$ $x_{A}, \eta(0)=y_{A}, \xi\left(T_{1}\right)=x_{B}, \eta\left(T_{1}\right)=y_{B}=h_{2}, \xi\left(T_{1}+0\right)=x_{C}, \eta\left(T_{1}+0\right)=y_{C}=$ $\left(1-\alpha_{2}\right) h_{2}, \xi\left(T_{1}+T_{2}\right)=x_{D}=h_{1}, \eta\left(T_{1}+T_{2}\right)=y_{D}, \xi\left(T_{1}+T_{2}+0\right)=x_{A}=x_{D}-\tau_{1}$ and $\eta\left(T_{1}+T_{2}+0\right)=\left(1+\alpha_{1}\right) y_{D}=y_{A}$ (see Figure $5(\mathrm{~b})$. Next, we prove the stability of the order- 2 periodic solution. 


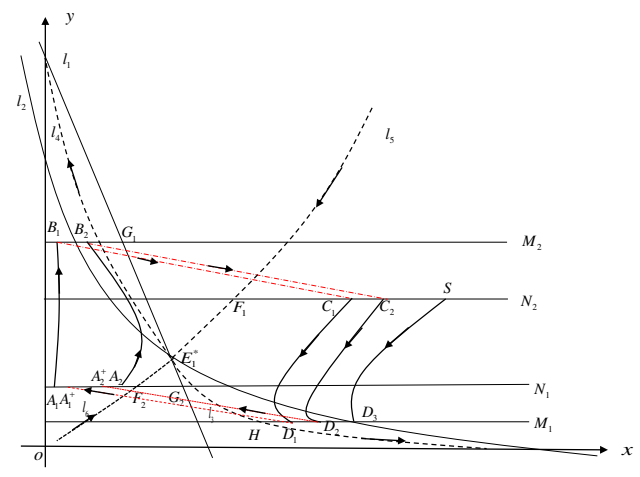

(a)

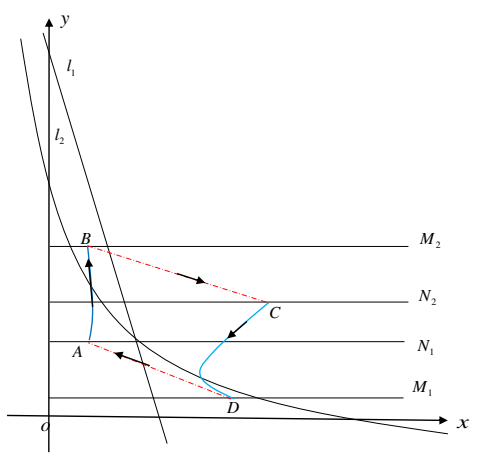

(b)

Fig. 5 Existence of the order-2 periodic solution of system (4).

Theorem 5 The order-2 periodic solution of system (4) is orbitally asymptotically stable if $\left|\Phi_{2}\right|<1$ holds, where

$$
\begin{aligned}
& \Phi_{2}=\Delta_{1} \Delta_{2} \frac{x_{B}\left(x_{A}+\tau_{1}\right)}{x_{A}\left(x_{B}+\tau_{2}\right)}, \\
& \Delta_{1}=\frac{\left(1-\alpha_{2}\right)\left(d-e\left(x_{B}+\tau_{2}\right)-f h_{2}\left(1-\alpha_{2}\right)\right)}{d-e x_{B}-f h_{2}}, \\
& \Delta_{2}=\frac{\left(1+\alpha_{1}\right)\left(d-e\left(x_{D}-\tau_{1}\right)-f h_{1}\left(1+\alpha_{1}\right)\right)}{\left(d-e x_{D}-f h_{1}\right)} .
\end{aligned}
$$

Proof Based on system (4), we have

$$
\begin{gathered}
P(x, y)=x\left(a-b x-\frac{c y}{1+y}\right), \quad Q(x, y)=y(d-e x-f y), \\
\frac{\partial P}{\partial x}=a-2 b x-c y, \frac{\partial Q}{\partial y}=d-e x-2 f y . \\
E_{1}(x, y)=\tau_{1}, \quad F_{1}(x, y)=-\alpha_{1} y, \quad \varphi_{1}(x, y)=y-h_{1}, \\
\frac{\partial E_{1}}{\partial x}=0, \quad \frac{\partial E_{1}}{\partial y}=0, \frac{\partial F_{1}}{\partial x}=0, \quad \frac{\partial F_{1}}{\partial y}=-\alpha_{2}, \quad \frac{\partial \varphi_{1}}{\partial x}=0, \quad \frac{\partial \varphi_{1}}{\partial y}=1, \\
E_{2}(x, y)=-\tau_{2}, \quad F_{2}(x, y)=\alpha_{2} y, \quad \varphi_{2}(x, y)=y-h_{2}, \quad \frac{\partial F_{2}}{\partial x}=0, \quad \frac{\partial \varphi_{2}}{\partial y}=1 . \\
\frac{\partial E_{2}}{\partial x}=0, \quad \frac{\partial E_{2}}{\partial y}=0, \frac{\partial F_{2}}{\partial x}=0, \quad \frac{\partial F_{2}}{\partial y}=\alpha_{2}, \quad
\end{gathered}
$$

By simple calculation,

$$
\begin{aligned}
\Delta_{1} & =\frac{P_{+}\left(\frac{\partial F_{1}}{\partial y} \frac{\partial \varphi_{1}}{\partial x}-\frac{\partial F_{1}}{\partial x} \frac{\partial \varphi_{1}}{\partial y}+\frac{\partial \varphi_{1}}{\partial x}\right)+Q_{+}\left(\frac{\partial E_{1}}{\partial x} \frac{\partial \varphi_{1}}{\partial y}-\frac{\partial E_{1}}{\partial y} \frac{\partial \varphi_{1}}{\partial x}+\frac{\partial \varphi_{1}}{\partial y}\right)}{P\left(\frac{\partial \varphi_{1}}{\partial x}\right)+Q\left(\frac{\partial \varphi_{1}}{\partial y}\right)} \\
& =\frac{Q_{+}\left(\xi\left(T_{1}+0\right), \eta\left(T_{1}+0\right)\right)}{P\left(\xi\left(T_{1}\right), \eta\left(T_{1}\right)\right)} \\
& =\frac{\left(1-\alpha_{2}\right)\left(d-e\left(x_{B}+\tau_{2}\right)-f h_{2}\left(1-\alpha_{2}\right)\right)}{d-e x_{B}-f h_{2}},
\end{aligned}
$$




$$
\begin{aligned}
\Delta_{2} & =\frac{P_{+}\left(\frac{\partial F_{2}}{\partial y} \frac{\partial \varphi_{2}}{\partial x}-\frac{\partial F_{2}}{\partial x} \frac{\partial \varphi_{1}}{\partial y}+\frac{\partial \varphi_{2}}{\partial x}\right)+Q_{+}\left(\frac{\partial E_{2}}{\partial x} \frac{\partial \varphi_{2}}{\partial y}-\frac{\partial E_{2}}{\partial y} \frac{\partial \varphi_{2}}{\partial x}+\frac{\partial \varphi_{2}}{\partial y}\right)}{P\left(\frac{\partial \varphi_{2}}{\partial x}\right)+Q\left(\frac{\partial \varphi_{2}}{\partial y}\right)} \\
& =\frac{Q_{+}\left(\xi\left(T_{1}+T_{2}+0\right), \eta\left(T_{1}+T_{2}+0\right)\right)}{Q\left(\xi\left(T_{1}+T_{2}\right), \eta\left(T_{1}+T_{2}\right)\right)} \\
& =\frac{\left(1+\alpha_{1}\right)\left(d-e\left(x_{D}-\tau_{1}\right)-f h_{1}\left(1+\alpha_{1}\right)\right)}{\left(d-e x_{D}-f h_{1}\right)},
\end{aligned}
$$

and

$$
\begin{aligned}
& \int_{0^{+}}^{T_{1}+T_{2}}\left(\frac{\partial P}{\partial x}+\frac{\partial Q}{\partial y}\right) d t=\int_{0^{+}}^{T_{1}+T_{2}} a-2 b x-\frac{c y}{1+y}+d-e x-2 f y d t \\
& =\int_{0^{+}}^{T_{1}} a-2 b x-\frac{c y}{1+y}+d-e x-2 f y d t+\int_{T_{1}}^{T_{1}+T_{2}} a-2 b x-\frac{c y}{1+y}+d-e x-2 f y d t \\
& =\int_{x_{A}}^{x_{B}} \frac{1}{x} d t+\int_{y_{A}}^{h_{2}} \frac{1}{y} d t+\int_{x_{C}}^{x_{D}} \frac{1}{x} d t+\int_{y_{C}}^{h_{1}} \frac{1}{y} d t-\int_{0^{+}}^{T_{1}+T_{2}} b x+f y d t
\end{aligned}
$$

Then we have

$$
\begin{aligned}
\mu_{2} & =\Delta_{1} \Delta_{2} \exp \left\{\int_{0^{+}}^{T_{1}+T_{2}}\left(\frac{\partial P}{\partial x}+\frac{\partial Q}{\partial y}\right) d t\right\} \\
& =\Delta_{1} \Delta_{2} \frac{x_{B}\left(x_{A}+\tau_{1}\right)}{x_{A}\left(x_{B}+\tau_{2}\right)} \exp \left\{-\int_{0^{+}}^{T_{1}+T_{2}} b x+f y d t\right\},
\end{aligned}
$$

and $\exp \left\{-\int_{0^{+}}^{T_{1}+T_{2}} b x+f y d t\right\}<1$. Define $\Phi_{2}=\Delta_{1} \Delta_{2} \frac{x_{B}\left(x_{A}+\tau_{1}\right)}{x_{A}\left(x_{B}+\tau_{2}\right)}$.

Thus, if $\left|\Phi_{2}\right|<1$, then $\left|\mu_{2}\right|<1$, and the order-2 periodic solution of system (4) is orbitally asymptotically stable.

\section{Examples and numerical simulations}

In this section, some numerical simulations will be presented to verify the theoretical results in the previous section. Different types of periodic solutions are performed with different control parameters.

Example 1 Select model parameters as follows: $a=0.45, b=0.7, c=0.9, d=$ $0.4, e=1, f=0.3$. If we just take unilateral control measure, then we have following two guanaco-sheep competitive systems with unilateral control:

$$
\left\{\begin{array}{l}
\frac{\mathrm{d} x}{\mathrm{dt}}=x\left(0.45-0.7 x-\frac{0.9 y}{1+y}\right), \\
\frac{\mathrm{d} y}{\mathrm{dt}}=y(0.4-x-0.3 y), \\
\left.\Delta x=-\tau_{1}, \Delta y=\alpha_{1} y\right\} \quad y=h_{1}, \\
x_{0}>0, y_{0}>h_{1} .
\end{array}\right.
$$


and

$$
\left\{\begin{array}{l}
\frac{\mathrm{d} x}{\mathrm{dt}}=x\left(0.45-0.7 x-\frac{0.9 y}{1+y}\right), \\
\frac{\mathrm{d} y}{\mathrm{dt}}=y(0.4-x-0.3 y), \\
\left.\Delta x=\tau_{2}, \Delta y=-\alpha_{2} y\right\} \quad y=h_{2}, \\
x_{0}>0, y_{0}<h_{2} .
\end{array}\right.
$$

In order to get the order-1 periodic solution of system (6), we select control parameters $h_{1}=0.15, \alpha_{1}=0.25$ and $\tau_{1}=0.02$. We find that the trajectory starting from the initial value $\left(x_{0}, y_{0}\right)=(0.4,0.5)$ will tend to be an order-1 periodic solution (see Figure 6). And Figure 6(d) is a partial enlargement of Figure $6(\mathrm{c})$.

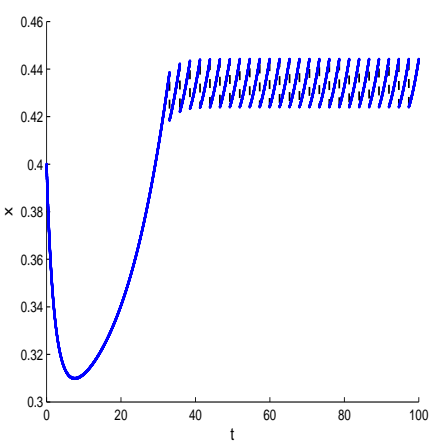

(a)

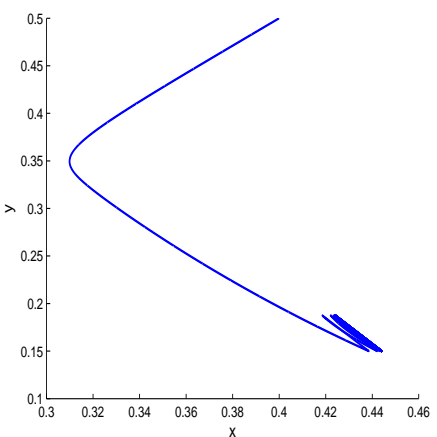

(c)

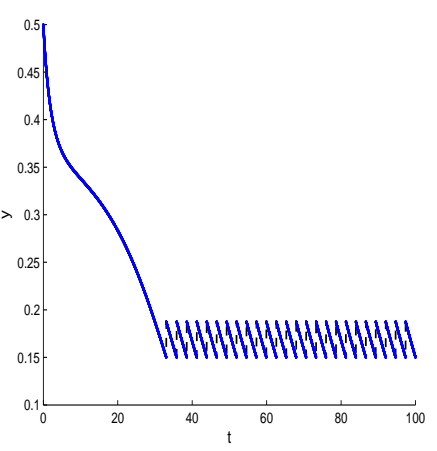

(b)

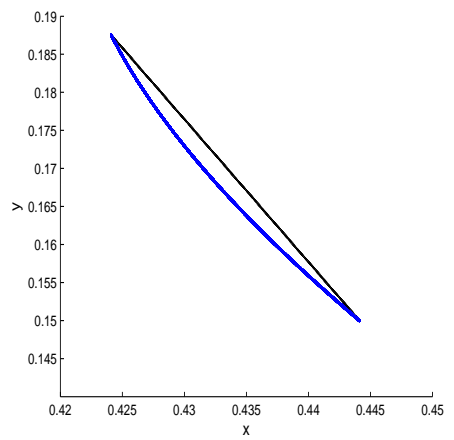

(d)

Fig. 6 Existence of the order-1 periodic solution of system [6].

We keep other parameters unchanged but $\tau_{1}=0.2$, the trajectory from the same initial value also tends to be an order-1 periodic solution (see Figure 7). 
Comparing Figures 6(d) and 7(c), we find that system [6] has different types of order-1 solution with different values of $\tau_{1}$.

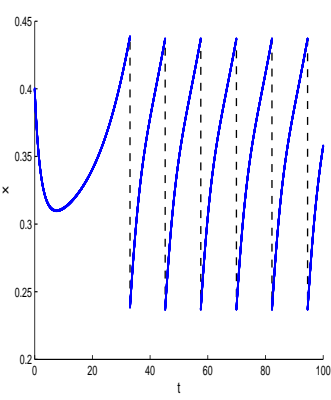

(a)

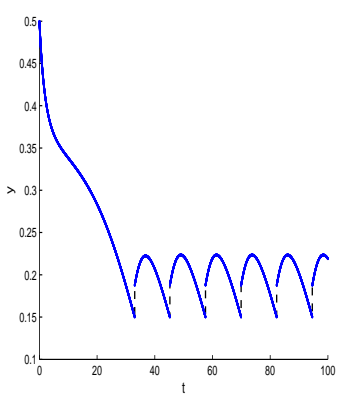

(b)

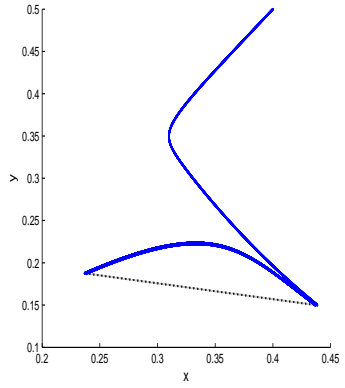

(c)

Fig. 7 Existence of the order-1 periodic solution of system 6 .

In order to get the order-1 periodic solution of system (7), we select control parameters: $h_{2}=0.75, \alpha_{2}=0.4$ and $\tau_{2}=0.12$. And we find that the trajectory starting from initial value $\left(x_{0}, y_{0}\right)=(0.2,0.5)$ tends to be an order- 1 periodic solution (see Figure 8).

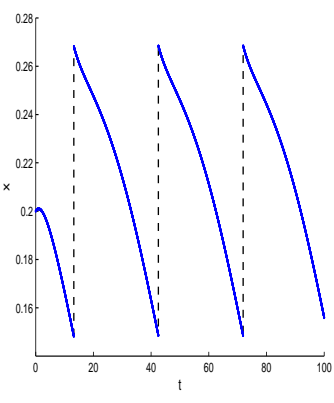

(a)

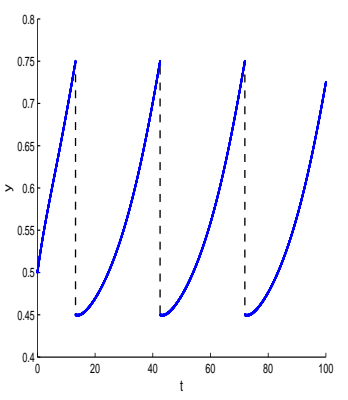

(b)

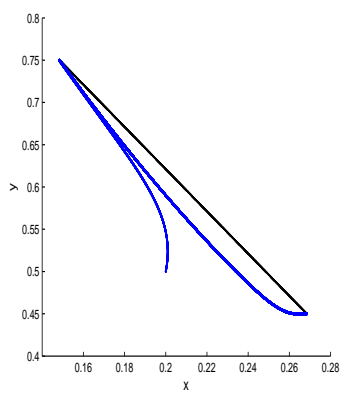

(c)

Fig. 8 Existence of the order-1 periodic solution of system 7

Keep other parameters unchanged, choosing the control parameter $\tau_{2}=0.05$, and we find the trajectory from same initial value also tends to be an order-1 periodic solution (see Figure 9). Comparing Figures 9(c) and 10(c), we find that system (7) has different types of order-1 solutions with different values of $\tau_{2}$. 


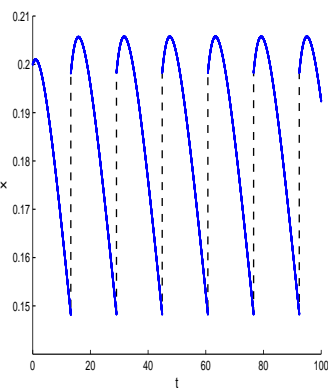

(a)

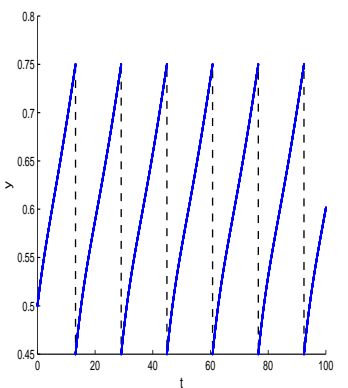

(b)

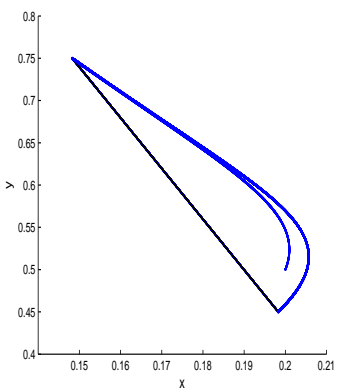

(c)

Fig. 9 Existence of the order-1 periodic solution of system 7

Example 2 In order to illustrate the existence of order-2 periodic solution of system (4) in section 3.3, by using the same model parameters, we get the following specific system

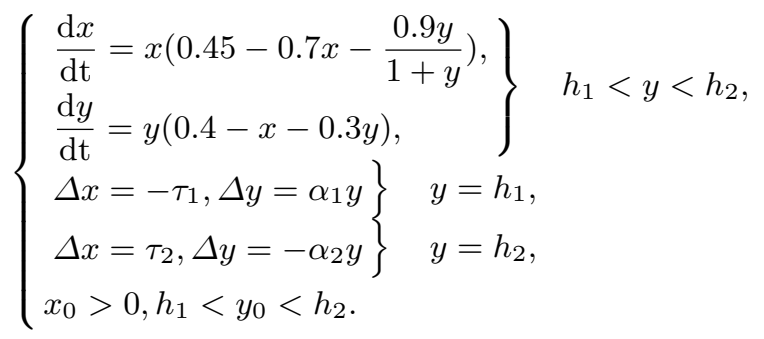

In order to get the order-2 periodic solution of system (8), we select control parameters $h_{1}=0.25, h_{2}=0.75, \alpha_{1}=0.6, \alpha_{2}=0.45, \tau_{1}=0.12$ and $\tau_{2}=0.25$. From the initial value $\left(x_{0}, y_{0}\right)=(0.35,0.3)$, And we find that the trajectory from initial value $\left(x_{0}, y_{0}\right)=(0.35,0.3)$ tends to be an order- 2 periodic solution (see Figure 10. Keep other parameters unchanged but $\tau_{1}=0.075$, and we find that the trajectory from the same initial value tends to be an order- 2 periodic solution (see Figure 11).

Example 3 To show the bifurcation diagram of system (4), we choose $\tau_{2} \in(0,0.9)$ as bifurcation parameter and $h_{1}=0.25, h_{2}=0.75, \alpha_{1}=0.6, \alpha_{2}=0.45, \tau_{1}=0.075$. From Figure 12, we can see that system (4) can exhibit order 1, 2, 3, and 4, periodic orbits with $\tau_{2} \in(0,0.9)$. More precisely, there exists an order-1 periodic orbit for $\tau_{2} \in(0,0.258)$ and then an order- 2 periodic solution bifurcates from the order-1 periodic solution at $\tau_{2} \approx 0.258$. And system (4) exists order-2 periodic orbit for $\tau_{2} \in(0.258,0.45)$ and order-3 periodic solution for $\tau_{2} \in(0.45,0.792)$. Similarly, system (4) appears order-3 periodic solution at $\tau_{2} \approx 0.45$ and order- 4 periodic solution at $\tau_{2} \approx 0.792$. Choose $\tau_{2}=0.79,0.8$ and keep other parameters fixed, we 


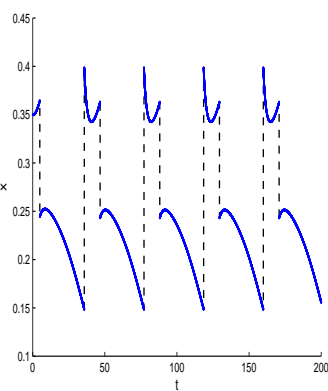

(a)

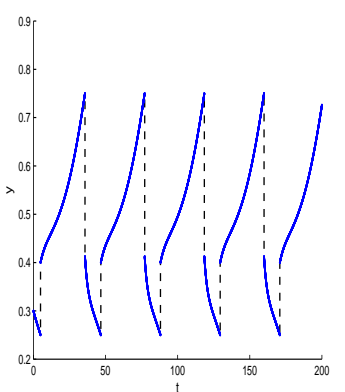

(b)

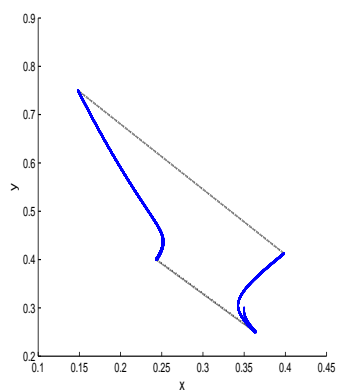

(c)

Fig. 10 Existence of the order-2 periodic solution of system 4.

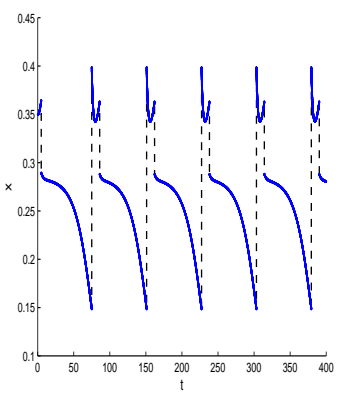

(a)

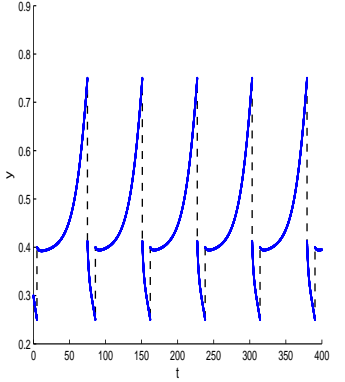

(b)

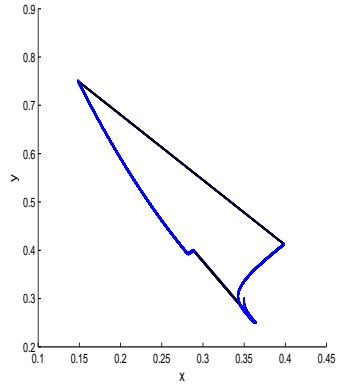

(c)

Fig. 11 Existence of the order-2 periodic solution of system (4).

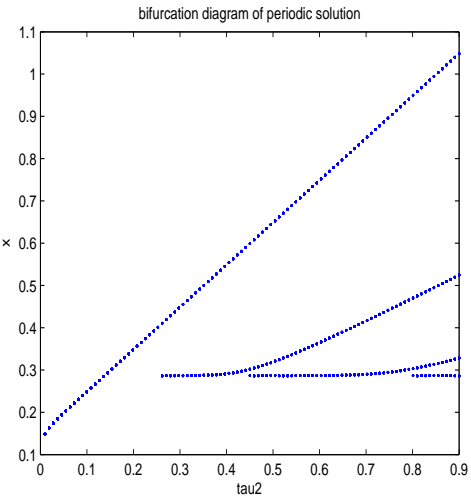

Fig. 12 Bifurcation diagram of system with $\tau_{2} \in(0,0.9)$.

show the existence of order-3 and order-4 periodic solution (see 13). Combing the Figures 12 and 13 , we find that system (4) has different types of periodic solutions 


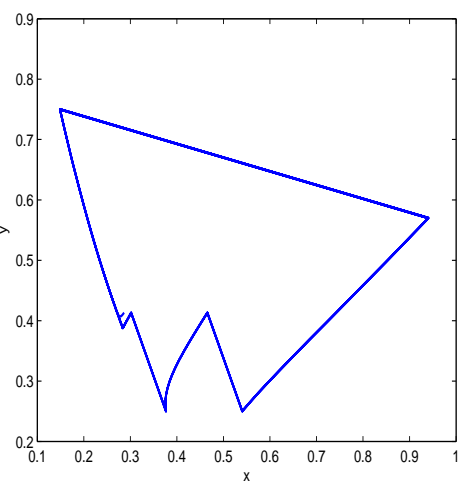

(a)

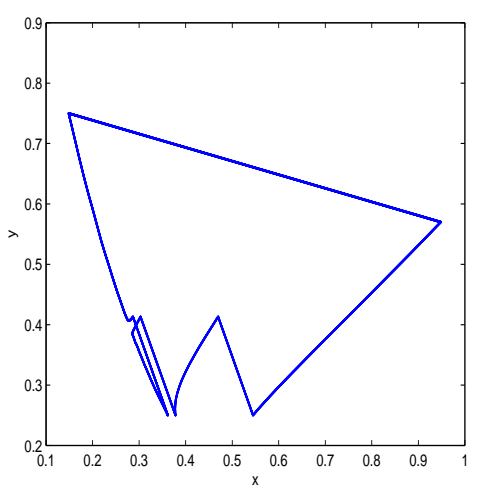

(b)

Fig. 13 Existence of the order-3 and order-4 periodic solution of system (4).

with the change of parameter $\tau_{2}$. And if we select $\tau_{1}, \alpha_{1}$ or $\alpha_{21}$ as bifurcation parameter, we have similar results, so omitted here.

\section{Conclusions}

In this paper, we develop and analyze a mathematical model for the management of overgrazing based on a guanaco-sheep competitive system with unilateral and bilateral control. And the case that the unique positive equilibrium $E_{1}^{*}$ of the basic ODE model is unstable is mainly discussed. More precisely, the existence of order1 and order-2 periodic solution is verified by the method of successor function. And the stability of order- 1 and order- 2 periodic solution is proved by applying the geometry theory and analogue of Poincaré criterion.

Numerical results show that if the control parameter $\alpha_{1}, \tau_{1}$ are given suitably, then system (2) exists an order-1 periodic solution. It means that guanaco and sheep species can be maintained periodically in certain level. And if we change the value of $\tau_{1}$ and keep other parameters unchanged, then system (2) also has an order-1 periodic solution. Thus, system (2) exhibits different types of order-1 periodic solution with different values of $\tau_{1}$. There exists similar results for system (3). In addition, if the control parameters $\alpha_{1}, \tau_{1}, \alpha_{2}, \tau_{2}$ are chosen suitably, then system (4) has an order-2 periodic solution. By drawing bifurcation diagram of periodic solution with $\tau_{2} \in(0,0.9)$, we find that system (4) exists high order periodic solution, such as order- 3 and order- 4 periodic solutions. 
Our study shows that if unilateral control measure is adopted, then system (2) or (3) has order-1 periodic solution, it means that population size of these two species changes periodically. For system (2), this unilateral control strategy can only protect farmers' interests, but it may not alleviate desertification caused by overgrazing. For system (3), this unilateral control strategy can alleviate desertification caused by overgrazing, but it does not guarantee farmers' benefits. Combining these two aspects, then bilateral control strategy is adopted, and the results show that the guanaco species can be protected and sheep species can be limited at a certain level. It means that the farmer's profits could be guaranteed and the desertification caused by overgrazing may be mitigated. Comparing the unilateral control and bilateral control strategies, our study encourages bilateral control rather than unilateral control for the management of sheep species.

\section{Acknowledgement}

Research is supported by the National Natural Science Foundation of China (No. 12071407).

\section{Data availability}

Data sharing not applicable to this article as no datasets were generated or analysed during the current study.

\section{Compliance with Ethical Standards}

\section{Conflict of interests}

The authors declare that there is no conflict of interests.

\section{Appendix}

In this section, we introduce some definitions and lemmas about the geometric theory of the semi-continuous dynamical system. 
Consider semi-continuous dynamical system

$$
\left\{\begin{array}{l}
\frac{\mathrm{d} x}{\mathrm{dt}}=P(x, y), \\
\frac{\mathrm{d} y}{\mathrm{dt}}=Q(x, y), \\
\Delta x=E(x, y), \quad \text { if } \quad M(x, y) \neq 0, \\
\Delta y=F(x, y),
\end{array}\right\} \quad \text { if } \quad M(x, y)=0,
$$

where $M(x, y)$ is called the impulsive set. Denote the impulsive map $\phi:(x, y) \rightarrow$ $(x+\Delta x, y+\Delta y)$, i.e. $N(x, y)=\phi(M(x, y))$. And if $(x, y) \notin M(x, y)$, the system develops under the regulation of $f(x, y)=\left(\frac{\mathrm{d} x}{\mathrm{dt}}=P(x, y), \frac{\mathrm{d} y}{\mathrm{dt}}=Q(x, y)\right)$, this part is similar to a continuous system.

Next, the definition of the successor function and periodic solution of system (4) are introduced.

Definition 1 For system (4), the two impulsive sets can be denoted by $M_{1}=$ $\left\{(x, y) \in R_{+}^{2} \mid y=h_{1}\right\}$ and $M_{2}=\left\{(x, y) \in R_{+}^{2} \mid y=h_{2}\right\}$, respectively. The corresponding impulsive mappings are defined as: $\phi_{1}:\left(x, h_{1}\right) \in M_{1} \longrightarrow\left(x^{+}, y^{+}\right)=$ $\left(x-\tau_{1},\left(1+\alpha_{1}\right) h_{1}\right)$ and $\phi_{2}:\left(x, h_{2}\right) \in M_{2} \longrightarrow\left(x^{+}, y^{+}\right)=\left(x+\tau_{2},\left(1-\alpha_{2}\right) h_{2}\right)$, while the Phase sets of the impulsive mappings are denoted by $N_{1}=\phi_{1}\left(M_{1}\right)$ and $N_{2}=\phi_{1}\left(M_{2}\right)$.

Definition 2 1. For a certain point $P_{1} \in N_{1}$, the trajectory from $P_{1}$ intersects with $M_{1}$ at point $Q_{1}$, then the impulsive mapping $\phi_{1}$ maps it to the phase point $Q_{1}^{+} \cdot Q_{1}^{+}$is called the order- 1 successor point of point $P_{1}$, and $G\left(P_{1}\right)=y_{Q_{1}^{+}}-y_{P_{1}}$ is called the successor function of point $P_{1}$. If $Q_{1}^{+}$coincides with $P_{1}$, then the trajectory $\widehat{P_{1} Q_{1}} \cup \overline{Q_{1} P_{1}}$ forms an order-1 periodic solution, as is shown in Figure $14(\mathrm{a})$

2. For a certain point $P_{2} \in N_{2}$, the trajectory from $P_{2}$ intersects with $M_{2}$ at point $Q_{2}$, then the impulsive mapping $\phi_{2}$ maps it to the phase point $Q_{2}^{+} \cdot Q_{2}^{+}$ is called the order- 1 successor point of $P_{2}$, and $G\left(P_{1}\right)=y_{Q_{1}^{+}}-y_{P_{1}}$ is called the successor function of point $P_{2}$. If $Q_{2}^{+}$coincides with $P_{2}$, then the trajectory $\widehat{P_{2} Q_{2}} \cup \overline{Q_{2} P_{2}}$ forms an order-1 periodic solution, as is shown in Figure 14(a)

3. For a certain point $P_{1} \in N_{2}$, the trajectory from $P_{2}$ intersects with $M_{1}$ at point $Q_{1}$, then the impulsive mapping $\phi_{1}$ maps it to the phase point $Q_{1}^{+} \in$ $N_{1}$. And, the trajectory from $Q_{1}^{+}$intersects with $M_{2}$ at point $S_{1}$, then the impulsive mapping $\phi_{2}$ maps it to the phase point $S_{1}^{+} \in N_{2} . S_{1}^{+}$is called order-2 successor point of $P_{1}$. If $S_{1}^{+}$coincides with $P_{1}$, then the trajectory $\widehat{P_{1} Q_{1}} \cup \overline{Q_{1} Q_{1}^{+}} \cup \widehat{Q_{1}^{+} S_{1}} \cup \overline{S_{1} P_{1}}$ forms an order-2 periodic solution, as is shown in Figure 14(b) Besides, point $S_{1}^{+}$is called the order-2 successor point of $P_{1}$, and $G\left(P_{1}\right)=y_{S_{1}^{+}}-y_{P_{1}}$ is called the order-2 successor function of point $P_{2}$. 


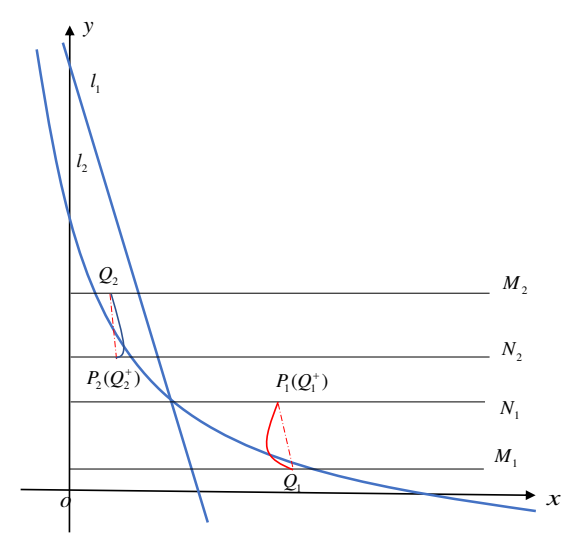

(a)

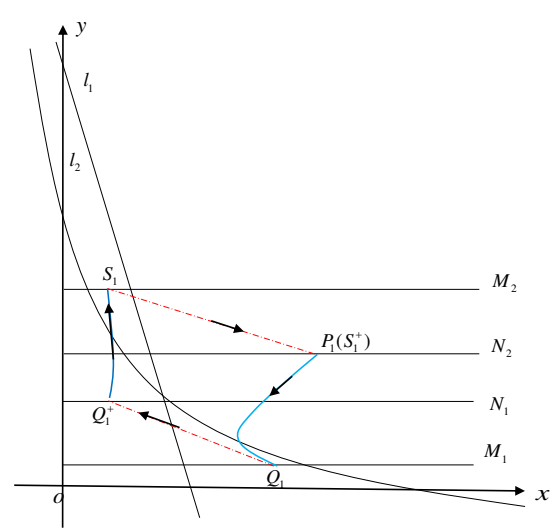

(b)

Fig. 14 Schematic diagram of the existence of order-1 and order-2 periodic solution.

\section{References}

1. J. Pedrana, A. Travaini, J. I. Zann, et al. Environmental factors influencing guanaco distribution and abundance in central Patagonia, Argentina. Wildlife Research, 2019, 46(1): $1-11$.

2. W. L. Franklin, Contrasting socioecologies of South Americas wild camelids: the vicuna and the guanaco. Advances in the study of mammalian behavior (JF Eisenberg and DG Kleiman, eds.). Advances in the study of mammalian behavior, 1983, 7: 573-629.

3. R. B. Baldi, P. Acebes, E. Cullar, et al. Lama guanicoe. IUCN Red List Threat Species, 2016.

4. W. L. Franklin, M. F. Bas, C. F. Bonacic, et al. Striving to manage Patagonia guanacos for sustained use in the grazing agroecosystems of southern Chile. Wildlife Society Bulletin, 1997, 25(1): 65-73.

5. C. Cunazza, S. Puig, L. Villalba, Situación actual del guanaco y su ambiente. In: Puig S (ed) Técnicas para el manejo del guanaco. IUCN, Buenos Aires, 1995, pp. 27-50

6. S. Puig, F. Videla, M. I. Cona, et al., Use of food availability by guanacos (Lama guanicoe) and livestock in Northern Patagonia (Mendoza, Argentina), Journal of Arid Environments, 2001, 47: 291-308.

7. R. Baldi, S. D. Albon, D. A. Eston, Guanacos and sheep: evidence for continuing competition in arid patagonia. Oecologia, 2001, 129: 561-570.

8. I. J. Gordon, What is the future for wild, large herbivores in human-modified agricultural landscapes? Wildlife Biology, 2009, 15(1): 1-9.

9. F. Hernandez, D. Corcoran, G. Graells, et al., Rancher perspectives of a livestock-wildlife conflict in Southern Chile. Rangelands, 2017, 39(2): 56-63.

10. M. F. Laguna, G. Abramsona, M. N. Kupermana, et al., Mathematical model of livestock and wildlife: Predation andcompetition under environmental disturbances. Ecological Modelling, 2015, 309: 110-117. 
11. Y. C. Daza C., M. F. Laguna, J. A. Monjeau, et al., Waves of desertification in a competitive ecosystem. Ecological Modelling, 2019, 396: 42-49.

12. A. Marino, V. Rodrguez, N. M. Schroeder, Wild guanacos as scapegoat for continued overgrazing by livestock across southern Patagonia. Journal of Applied Ecology, 2020, 57(12): 2393-2398.

13. F. R. Bharucha, K. A. Shankarnarayan, Effects of overgrazing on the grasslands of the western Ghats, India. Ecology 1958, 39(1): 152-153.

14. L. Chen, Pest control and geometric of semi continuous dynamical system. Journal of Beihua University (Natural Science), 2011, 12(1): 1-9.

15. H. Zhang, L. Chen, P. Georgescu, Impulsive control strategies for pest management. Journal of Biological Systems, 2007, 15: 235-260.

16. S. Tang, Y. Xiao, L. Chen, R. A. Cheke, Integrated pest management models and their dynamical behaviour. Bulletin of Mathematical Biology, 2005, 67: 115C135.

17. S. Tang, R. A. Cheke, State-dependent impulsive models of integrated pest management (IPM) strategies and their dynamic consequences. Journal of Mathematical Biology, 2005, $50(3): 257 \mathrm{C} 292$.

18. K. Sun, T. Zhang, Y. Tian, Dynamics analysis and control optimization of a pest management predator-prey model with an integrated control strategy, Applied Mathematics \& Computation, 2017, 292: 253-271.

19. J. Xu, Y. Tian, H. Guo, X. Song, Dynamical analysis of a pest management Leslie-Gower model with ratio-dependent functional response, Nonlinear Dynamics. 2018, 93(4): 705-720.

20. A. Singh, S. Gakkhar, State-dependent impulsive feedback control of a delayed preypredator system, Dynamics of Continuous Discrete \& Impulsive Systems, 2012, 19(2): 231249.

21. K. K. Su, C. Giphil, Lin-Fei N, State-dependent impulsive control strategies for a tumorimmune model, Discrete Dynamics in Nature and Society. 2016, 2016, 2979414(10 pages).

22. H. Guo, L. Chen, and X. Song, Qualitative analysis of impulsive state feedback control to an algae-fish system with bistable property, Applied Mathematics and Computation, 2015, 271: 905C922.

23. J. Fu and L. Chen, Modelling and qualitative analysis of water hyacinth ecological system with two state-dependent impulse controls, Complexity 2018, 2018, Article ID:4543976, 16 pages.

24. J. Xu, M. Huang, X. Song, Dynamical analysis of a two-species competitive system with state feedback impulsive control, International Journal of Biomathematics, 2020, 13(78), 2050007, 20pages.

25. M. Zhang, Y. Zhao, L. Chen, et al. State feedback impulsive modeling and dynamic analysis of ecological balance in aquaculture water with nutritional utilization rate, Applied Mathematics and Computation, 2020, 373, 125007.

26. M. Zhang, Y. Zhao, X. Song, Dynamics of bilateral control system with state feedback for price adjustment strategy, International Journal of Biomathematics, DOI: 10.1142/S1793524521500315, 2150031 (17 pages).

27. M. Huang, S. Liu, X. Song, et al., Dynamics of unilateral and bilateral control systems with state feedback for renewable resource management, Complexity, 2020, 2020, Article ID 9453941, 16 pages.

28. D. Tilman, R. M. May, C. L. Lehman, et al., Habitat destruction and the extinction debt. Nature, 1994, 371: 65-66.

29. S. Liu, L. Chen, G. Luo, Asymptotic behaviors of competitive Lotka-Volterra system with stage structure, Journal of Mathematical Analysis \& Applications. 2002, 271: 124-138. 